ThE AsTrophysiCAL Journal, 402:L53-L56, 1993 January 10

(C) 1993. The American Astronomical Society. All rights reserved. Printed in U.S.A.

\title{
METALLICITY MAPS OF THE GLOBULAR CLUSTER SYSTEM IN THE GALAXY
}

\author{
Emilio J. Alfaro \\ Instituto de Astrofísica de Andalucía, Apartado Correos 3004, Granada, 18080 Spain; and \\ Boston University, 725 Commonwealth Avenue, Boston, MA 02215 \\ JESÚs CABRERA-CAÑo \\ Instituto de Astrofísica de Andalucía, Apartado Correos 3004, Granada, 18080 Spain; and \\ Universidad de Sevilla, Apartado Correos 1065, Sevilla, 41080 Spain \\ AND \\ Antonio J. Delgado \\ Instituto de Astrofísica de Andulacía, Apartado Correos 3004, Granada, 18080 Spain \\ Received 1992 September 14; accepted 1992 October 23
}

\begin{abstract}
The spatial metallicity distribution in the Galaxy, as depicted by the globular cluster system, is analyzed. Two metallicity maps (on the $\omega-|Z|$ plane) corresponding to the halo and disk subsamples (Zinn 1985) have been obtained.

The resulting pictures reveal the existence of a highly structured abundance pattern for the two subsystems. Variations of the metallicity with $\omega$ as well as with $|Z|$ are clearly evidenced in both maps. However, the slope of these variations is very dependent on the direction chosen to estimate the gradient. This effect is particularly pronounced for the halo sample of globular clusters. Our results indicate that the metallicity distribution of the halo subsystem does not allow a simple description in terms of a single, direction independent gradient and that, possibly, this is the main cause of the long-standing controversy generated around the search for such a gradient.

Superposed on this large-scale structure is the clearly visible presence of several metallicity patches of variable size. The most striking of them is a low-metallicity spot (LMS) located at the solar galactocentric radius, which extends up to $6 \mathrm{kpc}$ away from the Galactic plane.
\end{abstract}

Subject headings: Galaxy: abundances - Galaxy: formation - Galaxy: halo - globular clusters: general

\section{INTRODUCTION}

Since the seminal paper of Eggen, Lynden-Bell, \& Sandage (1962), giving for the first time a global scenario for the formation and early evolution of our galaxy, much literature has been written about the role played in such a scheme by the presence or absence of an abundance gradient perpendicular to the Galactic plane. The existence and size of such abundance gradient is a sensitive test to the amount of dissipation which occurred during halo formation and, consequently, an important constraint on models on the formation of the Galaxy.

The available sample of globular clusters seems to be particularly well suited for the study of the metallicity distribution in the halo of our Galaxy. First, due to their brightness, we can reasonably assume that this sample is not strongly biased by lack of completeness. Second, the observational works performed by several authors in the last decade have greatly contributed to the establishment of a quite homogeneous internal metallicity scale for these objects (Zinn \& West 1984), and to the reliable determination of distances and metallicities for most of them (see Armandroff 1992, and references therein). In principle, this should allow an analysis of the metallicity spatial distribution with good prospects of success.

However, the search for and eventual evaluation of a metallicity gradient in the halo, based on the distribution of globular clusters, has had a controversial development in the last decades (see Searle \& Zinn 1978; Harris \& Canterna 1979; Pilachowski 1984; Zinn 1980, 1985; Armandroff 1992), and remains one of the basic unsettled questions in Galactic astronomy.

The description of the halo abundance distribution in terms of a one-dimensional metallicity gradient implies the assumption of some simplifying hypotheses about the actual distribution underlying the data. For example, the estimation of the $[\mathrm{Fe} / \mathrm{H}]$ variation with galactocentric distance rests on the postulate that the chemical pattern in the halo has spherical symmetry. Any departure in the data from the assumed model could give rise to contradictory results, otherwise very dependent on the sample properties. In this sense, the obtaining of a two-dimensional picture of the globular cluster metallicity distribution, based upon less restrictive geometrical constraints, should provide new insights on the actual abundance pattern in the Galaxy.

But, how to perform this task? We recall here a statement of Sandage (1990) referring to the remarkable similarity between astronomy and geology, as "sciences of the origins," in the sense that for both sciences "the key to the past lies in clues from the present." Here we take recourse not only to the geological strategy, but to the tactics. The technical problem we are facing consists in obtaining the two-dimensional distribution $|Z|-\omega$ of the metallicity ( $\omega$ is galactocentric distance projected on the Galactic plane, and $|Z|$ is absolute value of the height in respect to it) starting from a somewhat sparse sample of measurements. The close similarity of this problem with the task of evaluating and mapping natural resources in 
geology has been recently noted and stressed by CabreraCaño, Alfaro, \& Polvorinos (1990) and Alfaro, Cabrera-Caño, \& Delgado $(1991,1992)$ who have introduced and shown the power of the Kriging methodology in the treatment of astronomical problems.

In the present investigation our aim is to obtain the twodimensional $(\omega,|Z|)$ metallicity pattern for the globular cluster system in the Milky Way, having in mind a clarification of the chemical properties of the halo and disk subsystems (Zinn 1985). We leave aside, for the moment, discussion of important related matters, such as the connection between disk globular clusters and the subdwarf thick disk, or whether the halo subsystem might be considered as representative of the stellar halo population. This Letter has been structured in three sections. The second one briefly describes the source material and the methodology used in obtaining the metallicity maps. Last, the description and discussion of the main features appearing in these pictures are presented in $\S 3$.

\section{DATA AND METHODOLOGY}

\subsection{Data}

Our source material is the list of 93 globular clusters with reliable determinations of apparent magnitudes of horizontal branch stars, metallicities, and reddening values, compiled by Armandroff (1989). The number of objects with accurate measurements of these quantities increased in a later compilation up to 113 clusters (Armandroff 1992). However, out of these, six objects are located farther than $40 \mathrm{kpc}$ from the Galactic center, and eight between 20 and $40 \mathrm{kpc}$. This makes the density of clusters in the outer halo too low for the use of the Kriging method in delineating the metallicity map. We therefore restrict our analysis to galactocentric distances below 20 $\mathrm{kpc}$, where both samples are almost coincident, and use the list from 1989 including those values improved in the later compilation.

The absolute magnitude calibration of Liu \& Janes (1990) has been used to redetermine values for the heliocentric and galactocentric distances, adopting here a value of $\mathrm{Av} /$ $E(B-V)=3.2$, and $R_{\odot}=8.5 \mathrm{kpc}$. The calibration of Liu \& Janes (1990) shows a similar slope but differences of some tenths of a magnitude in the zero point with the theoretical calibration of Lee (1989). Our choice of the former was simply motivated by its observational nature.

The existence of two globular clusters subsystems, with different spatial, kinematic, and chemical properties (Zinn 1985), has been assumed. The separation line between the two groups is statistically well defined at $[\mathrm{Fe} / \mathrm{H}]=-0.8 \mathrm{dex}$ (Armandroff \& Zinn 1988), although a small number of overlapping objects could be expected (Armandroff 1992). With this subdivision the sample for the halo population contains 73 clusters, while the remaining 20 belong to the metal-rich disk subsystem. Most of the metallicity values display uncertainties below $0.20 \mathrm{dex}$, and the relative error in the determination of distances is about $20 \%$ on average.

\subsection{Method}

The basic problem in automatic cartography consists in computing some valid approximation of the target variable in points on the plane not belonging to the sampling set. From the large variety of algorithms able to perform this task we have chosen the Kriging technique as the more reliable one for this particular case. This method places the automatic cartog- raphy problem in the domain of the random process theory, and might be actually preferable when dealing with the analysis of natural phenomena (Alfaro et al. 1991). The mathematical bases of Kriging have been widely discussed in two previous papers (Cabrera-Caño et al. 1990; Alfaro et al. 1991), and we refer the reader to them for a better understanding of how this method does work.

The space of measurements is defined by the variables $|Z|, \omega$ and $[\mathrm{Fe} / \mathrm{H}]$. In order to avoid the lack of information in the borders $|Z|=0$ and $\omega=0$, we have built up a quadruple sample from the original one joining the $(|Z|, \omega),(|Z|,-\omega)$, $(-|Z|, \omega)$, and $(-|Z|,-\omega)$ quadrants. This is based upon the assumption that the metallicity pattern is not dependent on the Galactic azimuth and symmetric in respect to the Galactic plane.

An exploratory analysis of the globular cluster sample suggests the generalized covariance of order zero (representative of a noisy process) to be the best model accounting for the particular topology of the problem (see $\S 2.2 .8$ in Alfaro et al. 1991). This is equivalent to saying that we are dealing with the simplest case of Kriging, allowing an intuitive description of the procedure used for obtaining the maps. This procedure can be sketched as follows.

We select a grid of points on the plane, for which the metallicity has to be estimated, and evaluate the distances to the sample points for each node. These distances are further sorted in increasing order and classified in eight different files corresponding to the octants of the plane centered in the node. Later we set the number of nearest neighbors to be used in the estimation of the metallicity (12 in this case) and check whether they are distributed over five, at least, of the octants. If this is not the case, the node is rejected and no estimation of the metallicity in this point is performed. The mean, median, standard deviation, and mean error have been computed, in this way, for each node. The resulting maps were finally smoothed by means of a Gaussian filter.

This procedure was applied to the two samples of globular clusters corresponding to the disk and halo subsystems. The results are shown in Figures 1 and 2 (Plates L6 and L7), respectively.

\section{RESULTS AND DISCUSSION}

\subsection{Metallicity Maps}

The estimated metallicity maps are well defined inside the area limited by $|Z|=0.8-2 \mathrm{kpc}$ and $\omega=2-6 \mathrm{kpc}$ for the disk subsystem, and by $|Z|=1-17 \mathrm{kpc}$ and $\omega=1-19 \mathrm{kpc}$ for the halo sample of globular clusters. The mean error ranges between 0.02 and 0.06 dex for those clusters with [Fe/ $\mathrm{H}]>-0.8$ and 0.05 and 0.11 dex for the metal-poor subsample. These values are consistent with those determined by Armandroff $(1989,1992)$ for the two subsystems. Given that the $[\mathrm{Fe} / \mathrm{H}]$ and $R$ (galactocentric distance) observational errors are similar for the two subsamples, the larger mean error found for the metal-poor population seems to be indicative of an actual larger spread of metallicity for this subsystem.

A first glance to the metallicity maps displayed in Figures 1 and 2 reveals the existence of a highly structured abundance pattern for the two subsystems. This is even better marked for the disk population, where the $[\mathrm{Fe} / \mathrm{H}]$ isolines present a well defined elliptical shape. The minor axis runs parallel along the $|Z|$ coordinate, and the axis ratio is about $1: 3$. The metallicity map displayed in Figure 1 is, conceptually, similar to the 
PLATE L6

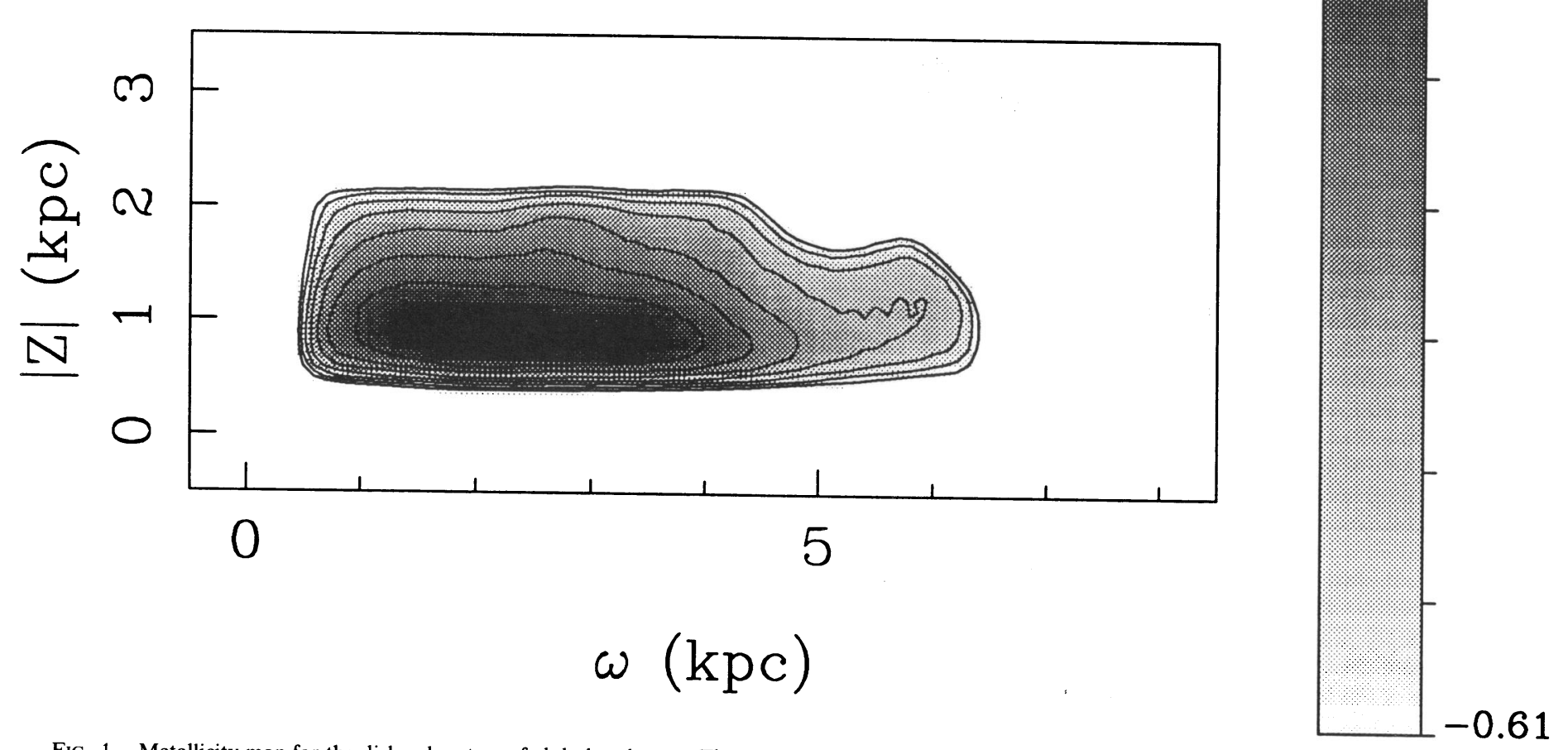

Fig. 1.- Metallicity map for the disk subsystem of globular clusters. The $\omega$ and $|Z|$ coordinates stand for the galactocentric distance projected on the Galactic plane and the absolute value of the height in respect to it, respectively. The isoline and gray scales are shown to the right of the map.

Alfaro, Cabrera-Caño, \& Delgado (see 402, L54) 


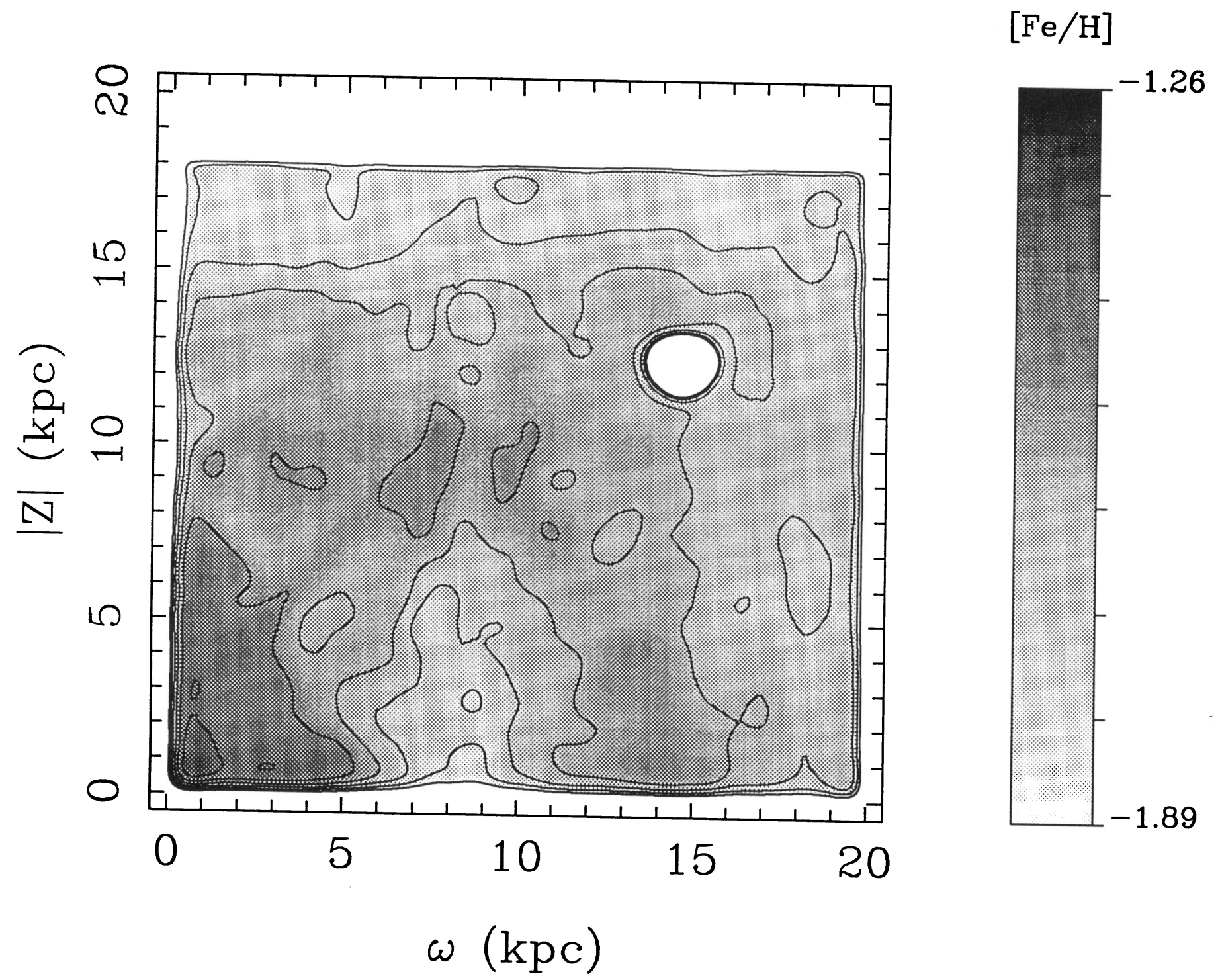

Fig. 2.- Metallicity map for the halo subsystem of globular clusters. Coordinates and scales as in Fig. 1. The white circular spot appearing in this map marks the region of the measurement space where the method fails to estimate the mean metallicity (see $\S 2.2$ in the text).

Alfaro, Cabrera-Caño, \& Delgado (see 402, L54) 
Figure 1 in Armandroff's (1989) paper, although the use of the Kriging method has allowed us to extract, in a more useful way, the spatial information contained in the data. The highly ordered structure present on this map suggests that the formation of the disk system was mainly governed by deterministic mechanisms, rather than by chaotic events.

The halo subsystem of globular clusters exhibits a more complicated design in metallicity. On one hand, the inner region of the Galaxy, $R<5 \mathrm{kpc}$, seems to display a high degree of symmetry. The same is true for the external regions, $R>14$ $\mathrm{kpc}$, where the $[\mathrm{Fe} / \mathrm{H}]$ isolines show a cylindrical pattern. However, superposed on this structure is the clearly visible presence of several metallicity patches of variable size. The most striking of them is a low-metallicity spot (LMS) in the vicinity of the solar galactocentric radius, which extends up to $6 \mathrm{kpc}$ away from the Galactic plane. This spot shows some peculiarities that differentiate it from the other patches of metallicity appearing on this map. First, the LMS covers a large area (about $15 \mathrm{kpc}^{2}$ ), whereas the other metallicity “islands" are restricted to smaller extents $\left(\approx 4 \mathrm{kpc}^{2}\right)$. Second, the variation of abundance across the LMS reaches up to 0.35 dex in $4 \mathrm{kpc}$, which in view of the estimated error of the metallicity ( $0.08 \mathrm{dex}$ for this region) leads to a signal-noise ratio larger than $\mathbf{4}$ for this structure. By contrast, the smaller patches show a difference in metallicity with the surrounding regions falling inside the error limits of the computed $[\mathrm{Fe} / \mathrm{H}]$ values. In order to corroborate the statistical significance of this morphological feature a more detailed test has been performed. Besides the errors in the determination of the metallicities, two other annoying factors could be envisaged as the possible cause of the LMS (1), the small number of sample points and (2), the distance errors. Bootstrap analysis (Nobelis 1990) provides a useful statistical tool to test the robustness of this structure (and hence of the Kriging method) to deliberate data editing. In order to perform this calculation we have selected a cut of the metallicity map going through the LMS at $|Z|=1 \mathrm{kpc}$. Further, we have generated 1000 random samples of 72 objects, where the spatial variable of each trial are the realization of a Gaussian process which mean is the actual sample value and, which dispersion is evaluated as the $20 \%$ of its distance to the Sun. The Kriging algorithm has then been rerun for every random sample and afterwards evaluated the mean and standard deviation of the Kriging values for 20 points along the $|Z|=1 \mathrm{kpc}$ axis. These results (continuous line and error bars) together with the profile computed from the original data set (dashed line) are displayed in Figure 3. The neat similarity between both profiles clearly evidences the reliability of the LMS feature and the power of the Kriging method to extract this kind of information. We therefore conclude that whereas the small patches of metallicity appearing on the halo metallicity map might be considered as statistical fluctuations originated in the whole estimation process, the LMS should be regarded as a real feature of the actual metallicity pattern in the Galaxy.

The large-scale variations of $[\mathrm{Fe} / \mathrm{H}]$ with spatial coordinates seems to suggest the concurrence of dissipative phenomena in the early phases of Galactic chemical evolution. However, the presence of the LMS indicates that some regions of the Galactic halo (at least one) have either undergone different evolutionary paths or evolved from different primordial abundances. A more detailed analysis of the kinematic, spatial, and chemical properties of the globular clusters forming up the LMS becomes necessary before proposing a reliable scenario for its origin.

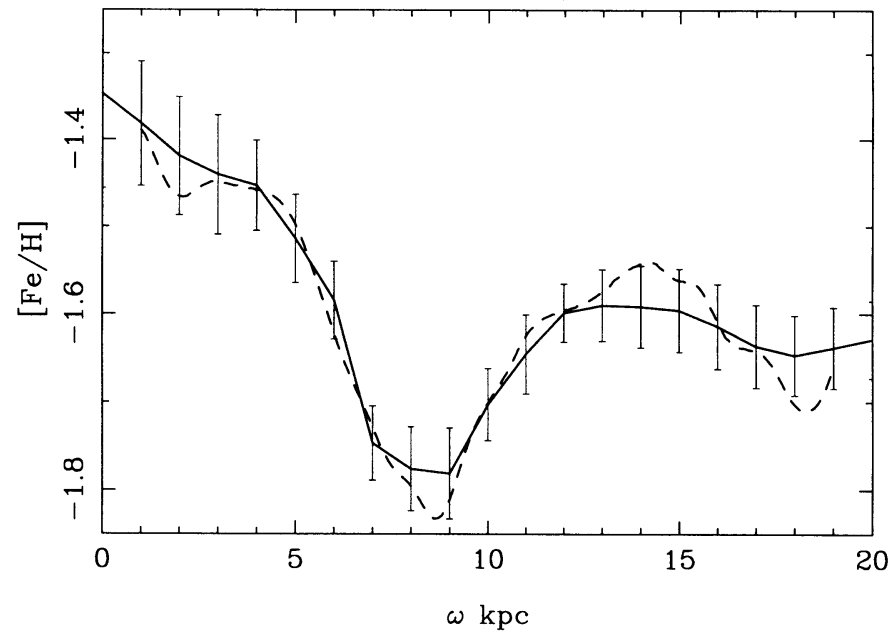

FIG. 3.-Bootstraped metallicity cut along the line $|Z|=1 \mathrm{kpc}$ (continuous line and error bars) together with the Kriging profile for the same axis (dashed line). The deep corresponding to the LMS is clearly visible in both metallicity profiles, supporting the statistical significance of this feature.

\subsection{Abundance Gradients}

We have selected directions across the $(\omega,|Z|)$ plane in order to quantify the variations of metallicity with the spatial variables. Figure 4 displays the abundance gradients along the lines $|Z|=1 \mathrm{kpc}$ (top) and $\omega=2 \mathrm{kpc}$ (bottom) for the metalrich population of globular clusters. The error bars shown in these plots stand for the mean errors computed by the procedure outlined in $\S 2$.2. Two weak, but statistically significant, gradients can be observed in Figure 4. A linear fit to these plots
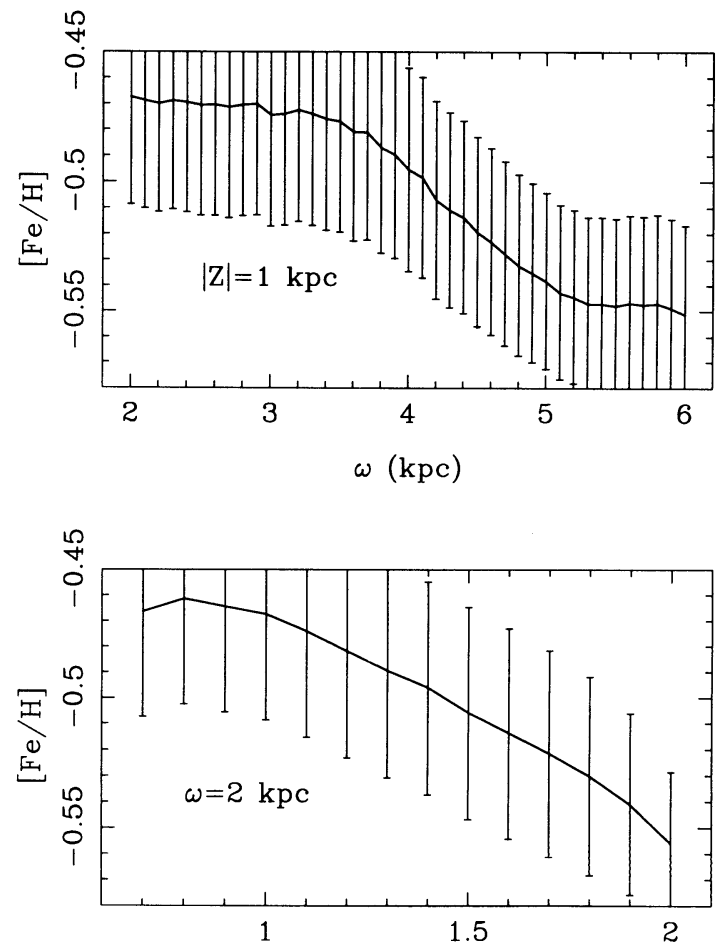

$|\mathrm{Z}|(\mathrm{kpc})$

Fig. 4.- Variation of the metallicity $([\mathrm{Fe} / \mathrm{H}])$ with $\omega$ along $|Z|=1 \mathrm{kpc}$ (top) and with $|Z|$ along the axis $\omega=2 \mathrm{kpc}$ (bottom), for the disk subsystem of globular clusters. The error bars are representative of the mean error computed as explained in the text. 


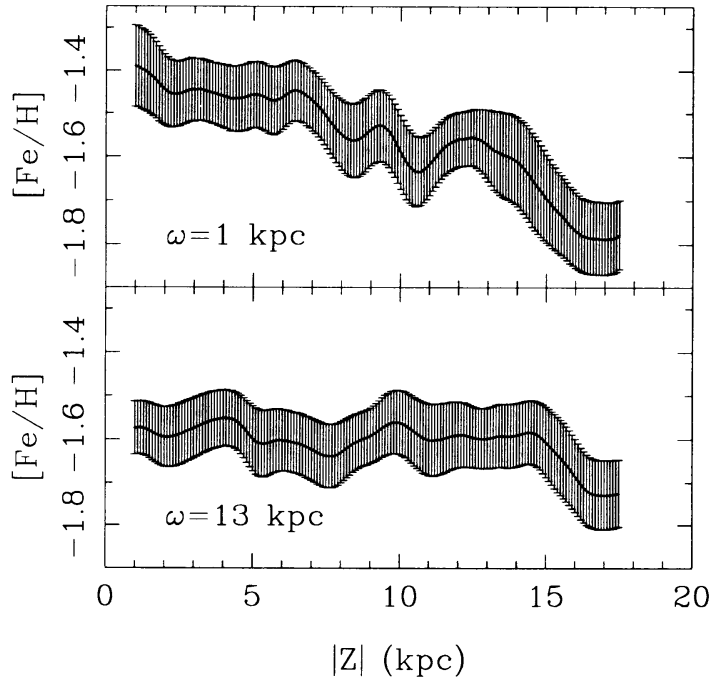

FIG. 5.-Variation of the metallicity $([\mathrm{Fe} / \mathrm{H}])$ with $|Z|$ along the axes $\omega=1 \mathrm{kpc}($ top $)$ and $\omega=13 \mathrm{kpc}$ (bottom) for the halo subsystem of globular clusters.

yields values for the slope of $(\Delta[\mathrm{Fe} / \mathrm{H}]) / \Delta \omega=-0.026 \pm 0.001$ $\operatorname{dex~kpc}{ }^{-1}$ and $(\Delta[\mathrm{Fe} / \mathrm{H}]) / \Delta|Z|=-0.072 \pm 0.004 \mathrm{dex} \mathrm{kpc}^{-1}$, in good agreement with those values estimated by Armandroff (1989) for the same subsystem.

A look at the metallicity map of the halo subsystem evidences that the variations of $[\mathrm{Fe} / \mathrm{H}]$ with $|Z|$ are strongly dependent on the $\omega$-value chosen to perform the cut. This is better visualized in Figure 5 where two slices of the metallicity matrix along the lines $\omega=1$ and $13 \mathrm{kpc}$ are shown. A clear abundance gradient with $|Z|$ is patent in this plot $(\omega=1 \mathrm{kpc})$, whereas for the $\omega=13 \mathrm{kpc}$ axis, the metallicity oscillates around a mean value $([\mathrm{Fe} / \mathrm{H}]=-1.59)$ and drops suddenly at $|Z|$ larger than $15 \mathrm{kpc}$.

In the same way, the abundance gradient with $\omega$ has been estimated for the directions $|Z|=1 \mathrm{kpc}$ and $|Z|=10 \mathrm{kpc}$ (Fig. 6). For $|Z|=1$, the $[\mathrm{Fe} / \mathrm{H}]$ versus $\omega$ profile is mainly governed by the presence of the LMS. The metallicity drops from $[\mathrm{Fe} / \mathrm{H}] \approx-1.39$ at $\omega=1$ down to $[\mathrm{Fe} / \mathrm{H}] \approx-1.83$ at $\omega=8.5 \mathrm{kpc}$. A linear least-squares fit to this segment yields a formal gradient with $\omega$ of $-0.058 \pm 0.003 \mathrm{dex} \mathrm{kpc}{ }^{-1}$. As a general rule the slope of the $[\mathrm{Fe} / \mathrm{H}]$ variation with $|Z|$ as well as with $\omega$ decreases as $\omega$ and $|Z|$ increase, except for those Galactic regions covered by the LMS where the behavior of the metallicity pattern is far from linear.

These results indicate (1) that the metallicity distribution pattern of the halo subsystem (overall, the presence of the LMS) does not allow a simple description in terms of a unique, averaged, abundance gradient along some particular

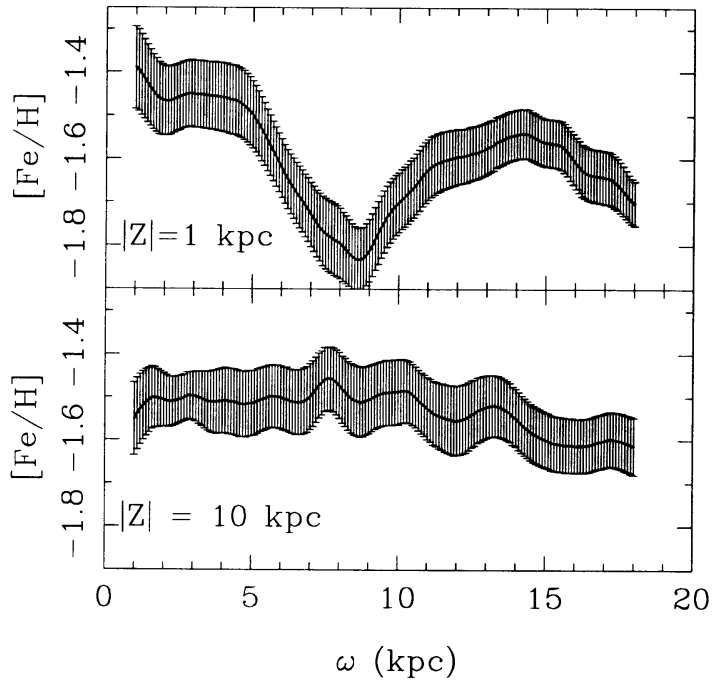

FIG. 6.-Variation of the metallicity $([\mathrm{Fe} / \mathrm{H}])$ with $\omega$ along the axes $|Z|=1 \mathrm{kpc}($ top $)$ and $|Z|=13 \mathrm{kpc}$ (bottom) for the halo subsystem of globular clusters.

direction and (2) that possibly, this is the main cause of the long-standing controversy generated by the search for such a gradient. As an exercise, we have estimated the variations of $[\mathrm{Fe} / \mathrm{H}]$ with $|Z|$ averaging over the whole range of $\omega$. The profile obtained is very similar, within the errors, to that obtained by Armandroff (1992) for the same population. This suggests that the existence of a gradient is not clearly detected in this case, simply because the internal structure of the metallicity distribution was hidden by the methodology used.

To sum up, the symmetry displayed by the $[\mathrm{Fe} / \mathrm{H}]$ isolines and the existence of weak but significant abundance gradients lead us to conclude that large-scale dissipative phenomena have played an important role in the formation of the disk and halo subsystems of globular clusters. However, the presence of the LMS opens new questions and imposes new observational constraints on the scenarios envisaged to draw the cosmogony of the Galaxy.

We are grateful to T. E. Armandroff for supplying a machine-readable version of the list of clusters used in this study. Special thanks are given to J. L. Gómez for his help in the preparation of Figures 1 and 2. We thank Ken Janes and an anonymous referee for their comments and suggestions which have improved the first version of this Letter. Financial support from DGICYT (Spain) through a sabbatical grant is acknowledged by E. J. A. This research was partially financed by the Consejería de Educación y Ciencia, Junta de Andalucía, Spain.
Alfaro, E. J., Cabrera-Caño, J., \& Delgado, A. J. 1991, ApJ, 378, 106 1992 ApJ 399,576

Armandroff, T. E. 1989, AJ, 97, 375

Armandroff, T. E. 1989, AJ,

Armandroff, T. E., \& Zinn, R. 1988, AJ, 96, 92

Cabrera-Caño, J., Alfaro, E. J., \& Polvorinos, A. 1990, in Errors, Bias, and

Uncertainties in Astronomy, ed. F. Murtagh \& C. Jascheck (Cambridge: Cambridge Univ. Press), 101

Eggen, O. J., Lynden-Bell, D., \& Sandage, A. 1962, ApJ, 136, 748

Harris, W. E., \& Canterna, R. 1979, ApJ, 231, L19

\section{REFERENCES}

Lee, Y.-W. 1989, Ph.D thesis, Yale Univ.

Liu, T., \& Janes, K. A. 1990, ApJ, 354, 273

Nobelis, Ph. P. 1990, in Errors, Bias, and Uncertainties in Astronomy, ed

F. Murtagh \& C. Jascheck (Cambridge: Cambridge Univ. Press), 89

Pilachowski, C. A. 1984, ApJ, 281, 614

Sandage, A. 1990, JRASC, 84, 70

Searle, L., \& Zinn, R. 1978, ApJ, 225, 357

Zinn, R. 1980, ApJ, 241, 602 1985, ApJ, 293, 424

Zinn, R., \& West, M. J. 1984, ApJS, 55, 45 